\title{
Labyrinthe
}

$24 \mid 2006$ (2)

Faut-il être postcolonial?

\section{Maitrise et déformation : les lumières diffractées}

\section{Anthony Mangeon}

\section{OpenEdition}

\section{Journals}

Édition électronique

URL : http://journals.openedition.org/labyrinthe/1249

DOI : $10.4000 /$ labyrinthe. 1249

ISSN : 1950-6031

Éditeur

Hermann

Édition imprimée

Date de publication : 15 juin 2006

Pagination : 63-83

ISBN : 2-9526131-1-7

Référence électronique

Anthony Mangeon, « Maitrise et déformation : les lumières diffractées », Labyrinthe [En ligne], 24 | 2006

(2), mis en ligne le 24 juillet 2008, consulté le 21 avril 2019. URL : http://journals.openedition.org/ labyrinthe/1249; DOI : 10.4000/labyrinthe.1249 


\title{
MAÎTRISE ET DÉFORMATION : LES LUMIÈRES DIFFRACTÉES*
}

\author{
Anthony MANGEON \\ anthony.mangeon@club-internet.fr
}

\begin{abstract}
À notre époque souvent qualifiée de «postmoderne » ou de «postcoloniale », l'heure n'est plus aux grands récits, mais assurément aux grands procès, et celui de l'Occident n'en finit plus d'être instruit. À juste titre, sans aucun doute. Dans ses excès militaires et culturels, la domination qu'exerça l'Europe sur l'Amérique, les Caraïbes, puis, lors de sa seconde expansion coloniale, sur l'Afrique et une large partie de l'Asie, parut souvent et reste encore injustifiable, puisqu'en pleine contradiction avec l'humanisme et les idéaux des Lumières qui étaient alors défendus : périssent donc nos colonies plutôt qu'un principe, la formule vaut aujourd'hui comme hier. La critique marxiste, qui fut longtemps à l'avant-garde des mouvements anticolonialistes, n'a cependant point suffi à rendre intelligible cette domination dans ses multiples contradictions: en présentant l'impérialisme comme un ultime avatar du capitalisme, elle fit en effet primer la dimension économique et matérielle, sans tenir suffisamment compte de la superstructure idéologique et de l'influence que cette dernière pouvait, en retour, exercer sur l'élaboration de l'infrastructure, puis sur la relation aux autres cultures; en donnant priorité au matérialisme historique, elle a négligé l'importance des idéalismes missionnaires et humanitaires dans la puissance d'expansion européenne ${ }^{1}$. Or, il ne s'est pas seulement agi d'une exploitation économique et commerciale: l'Europe a également porté un regard sur les peuples qu'elle a colonisés, elle les a représentés, voire exhibés; elle a parlé en leur nom, s'est prononcé sur leur statut, et ce faisant elle a souvent trahi
\end{abstract}

\footnotetext{
* Ce texte a bénéficié de la lecture et des commentaires de Viviane Azarian, Laurent Dubreuil, Frédéric Mambenga-Ylagou, Renaud Pasquier et la rédaction de Labyrinthe. Que tous en soient ici vivement remerciés.

1. Voir notamment Aimé Césaire, Discours sur le colonialisme [1955], Paris, Présence africaine, 1994; Franz Fanon, Les Damnés de la terre [1961], Paris, Gallimard, Folio/Essais, 1996; Walter Rodney, How Europe Underdeveloped Africa [1972], Washington, Howard University Press, 1982.
} 
l'universalisme religieux, juridique, politique qu'elle proclamait, proposait, ou prétendait incarner et diffuser. C'est donc afin de « renouveler l'étude des relations entre l'Europe et le monde, au-delà du discours marxisant » qu'une nouvelle démarche critique, « l'approche postcoloniale», aurait fait de sa nécessité vertu, et s'attacherait aujourd'hui à montrer que « les représentations ont de nombreuses incidences, non seulement dans le champ des images, mais aussi dans celui des décisions économiques et politiques ${ }^{2} »$.

Dans les pages qui suivent, je retracerai la genèse des problématiques postcoloniales dans les domaines orientalistes et africanistes, afin d'exposer ensuite comment leur importation en France, dans le champ d'une certaine historiographie, s'apparente plus à un détournement qu'à une appropriation critique; enfin, je voudrais soumettre l'approche postcoloniale elle-même à l'exigence archéologique et généalogique qui la sous-tend, pour montrer comment un grand nombre de ses problématiques et postures critiques furent notamment préfigurées par la tradition intellectuelle noire, encore trop largement méconnue en France.

\section{Les émules de Foucault}

La domination politique, culturelle, économique de l'Europe fut étroitement liée à une domination symbolique, c'est-à-dire à l'existence d'un savoir: préexistant, le plus souvent, à la rencontre (voir l'autre comme sauvage ou primitif, c'était en effet le reconnaître ainsi, notamment à partir des textes des Anciens) ${ }^{3}$, ce savoir fut en retour, dans ses développements, étroitement associé à la perpétuation du pouvoir. Étudier les modalités de cette imbrication (savoir/pouvoir) a donc constitué, au siècle dernier, un des axes majeurs dans l'analyse des relations du monde européen aux autres cultures; et l'un des thèmes dominants de la recherche en sciences humaines, à partir des années 1960, fut très précisément la dénonciation des liens entre la constitution des sciences humaines et l'impérialisme économique, politique, culturel des puissances européennes. Cette critique s'est notamment nourrie du brillant

2. Françoise Vergès, « Pour une lecture postcoloniale de Césaire », dans Aimé Césaire, Nègre je suis, Nègre je resterai, entretiens avec Françoise Vergès, Paris, Albin Michel, 2006, p. 73.

3. Gérard Lenclud, «Quand voir, c'est reconnaitre. Les récits de voyage et le regard anthropologique », Enquête, 1, Les terrains de l'enquête, Marseille, Parenthèses, 1995. 
réquisitoire qu'avait formulé Michel Foucault en exposant, dans Les Mots et les Choses (1966) ${ }^{4}$, l'indexation des sciences humaines et sociales sur des disciplines empiriques telles que la biologie ou l'économie. La démarche archéologique de Foucault, qui proposait d'étudier les rapports entre des « formations discursives », les règles qui les régissent, et « des domaines non discursifs (institutions, événements politiques, pratiques et processus économiques) $)^{5} »$, suscita rapidement de nombreux émules outre-Atlantique. La parution de L'Orientalisme (1978) a rétrospectivement permis de dater l'émergence des « études postcoloniales ${ }^{6}$ », mais l'on pourrait ajouter au nom d'Edward Said ceux de Christopher Miller, professeur à Yale, et de Valentin-Yves Mudimbe, écrivain et universitaire de l'ex-Zaïre naturalisé américain: leurs ouvrages respectifs, Blank Darkness (1985), The Invention of Africa (1988) et The Idea of Africa (1994), analysent comment « le terme d'Afrique, qui semble référentiel, est tout aussi chargé d'investissements rhétoriques que l'Orient si évidemment fictif ${ }^{7}$ », et ils étudient « la Bibliothèque coloniale » qui s'est ainsi progressivement constituée, depuis les premiers récits de voyageurs et d'explorateurs jusqu'aux textes des anthropologues et écrivains contemporains. «Cette tradition discursive provenant d'un seul lieu épistémologique ${ }^{8}$ » aurait «inventé» une représentation univoque de l'Afrique, continent primitif où règnent la nature et la bestialité, y compris dans l'ordre humain dominé par les seuls instincts vitaux et sexuels. Les analyses de Mudimbe, qui interprète "l'invention européenne de l'Afrique » comme un processus d' « idéation de soi $^{9}$ », ont récemment reçu un retentissant écho dans les textes d'Achille Mbembe, un historien d'origine camerounaise ${ }^{10}$. Pour se prémunir contre les

\footnotetext{
4. Paris, Gallimard, collection TEL, 1995 [désormais abrégé $M C$ ].

5. Michel Foucault, L'Archéologie du savoir, Paris, Gallimard, 1969, p. 212.

6. Traduction française par Catherine Malamoud, Paris, Seuil, 1980 [désormais abrégé $O$ ]. Voir supra, l'entretien avec Anne Berger.

7. Christopher Miller, Blank Darkness, Africaniste Discourse in French, Chicago, University of Chicago Press, 1985, p. 14.

8. V. Y. Mudimbe, The Idea of Africa, Bloomington, Indiana University Press, 1994, p. XV.

9. V. Y. Mudimbe, The Invention of Africa, Bloomington, Indiana University Press, 1988, p. 71 [désormais IA] ; Tales of Faith, Religion as Political Performance in Central Africa, Londres, The Athlone Press, 1997, p. 17-25 [désormais abrégé $T F$ ].

10. De la postcolonie, essai sur l'imagination politique dans l'Afrique contemporaine [2000], Paris, Karthala, 2005 [désormais $D P$ ] ; « La République et l'impensé de la "race" », dans Nicolas Bancel, Pascal Blanchard et Sandrine Lemaire (éd.), La Fracture coloniale. La société française au prisme de l' héritage colonial, Paris, La Découverte, 2005.
} 
critiques qui pouvaient, en retour, les accuser de verser dans un certain «occidentalisme», les penseurs postcoloniaux ont développé deux démarches complémentaires : la première consista à pratiquer la nuance et la vigilance épistémologique, et la seconde, à favoriser et à valoriser les multiples domaines d'intersection que la confrontation Afrique/ Occident avait inévitablement engendrés.

Mudimbe reconnaît en effet certains changements d'orientation et certaines réévaluations qualitatives dans le rapport des Européens aux cultures non-occidentales. À compter des années 1920, on peut observer dans l'ethnographie africaniste initiée en France par Maurice Delafosse, dans l'anthropologie culturelle gouvernée aux États-Unis par Franz Boas et Melville Herskovits, ainsi que dans l'anthropologie sociale pilotée, en Angleterre, par Malinowski et son fonctionnalisme de nouvelles approches qui se caractérisent par un plus grand respect des cultures indigènes ${ }^{11}$. Les travaux de ces divers penseurs contribuent à saper les préjugés évolutionnistes, au bénéfice d'une plus grande attention pour les logiques et les normes propres à chaque « individualité culturelle» $(T F$, ix et 40). C'est précisément une telle démarche qu'Achille Mbembe appelle encore de ses vœux en déplorant aujourd'hui que

les critères que les agents africains reconnaissent comme valides et les raisons qu'ils échangent à l'intérieur de leurs propres pratiques instituées de rationalité [soient], aux yeux de beaucoup, sans valeur. Ce qui vaut pour les agents africains en tant que raisons d'agir, ce que leur prétention à agir selon une raison implique comme prétention générale à avoir raison, ce qui rend leur action intelligible à leurs propres yeux : rien de tout cela ne compte guère aux yeux des analystes $(D P, 18)$.

Conscient que la pensée occidentale n'est point uniforme, mais traversée de régimes conceptuels concurrentiels, Mudimbe propose de dépasser les oppositions statiques et binaires - entre Afrique et Occident, tradition et modernité - en approchant désormais toute tradition, qu'elle soit historique, culturelle, épistémologique, en termes de « discontinuités inscrites au cœur d'une continuation dynamique et de reconversion possible de choses héritées » (IA, 189). Mbembe partage une même volonté de privilégier la «continuation de discontinuités», intérieures

11. Mudimbe, $I A, 35,47,72,76$ et 136 . 
autant qu'extérieures à l'histoire de la pensée occidentale, dans la mesure où elles en altèrent profondément l'hégémonie.

Nous cherchons désormais à écrire l'Afrique dans un contexte caractérisé, plus que par le passé, par la reconnaissance de la pluralité des savoirs. La vision de la théorie sociale $[\ldots]$ a subi, au cours des dernières années du $\mathrm{XX}^{\mathrm{e}}$ siècle, de profondes remises en cause. Il y a eu d'abord l'éclatement de l'univers des sciences naturelles et le recul du modèle newtonien. [...] L'une des conséquences de cet éclatement est la mise en cause des raisonnements déterministes aussi bien dans l'élaboration des théories de la société que dans la définition des théories de l'action individuelle et sociale. Cette remise en cause est allée de pair avec trois processus dont l'importance ne saurait être sous-estimée. Le premier est le rejet des philosophies totalisantes de l'histoire et la reconnaissance de la contingence du social et de l'historique. Le deuxième est l'affirmation de la multiplicité des mondes et des formes de la vie. Le troisième est la reconnaissance de formes de savoir distinctes du savoir dit scientifique. [...] L'héritage du rationalisme occidental ne vaut plus de manière incontestée. L'idée de rompre avec les Lumières et ce qu'elles ont promu a, de fait, favorisé le développement d'une compréhension décentrée du monde. Hors du cadre exclusif de la modernité occidentale, d'autres formations de la conscience universelle émergent, même si ce procès reste profondément lié aux conditions de l'impérialisme colonial et du capitalisme moderne » $(D P, 26-27)$.

L'un des premiers effets - et non le moindre! - de ces nouvelles orientations est de rendre audible la prise de parole des premiers écrivains et penseurs noirs dans notre univers intellectuel ${ }^{12}$. Bien au fait de l'histoire littéraire francophone, Mudimbe sait également reconnaître l'influence déterminante des Africains-Américains dans cette genèse d'un «nouveau discours »: un chapitre de son Invention of Africa examine notamment «l'héritage et les questions d'Edward Wilmot Blyden » ou la préfiguration, dans ses textes, des principales thèses de la négritude.

S'ils s'inspirent ouvertement de Michel Foucault, les ouvrages de Said, Mudimbe et Mbembe s'éloignent toutefois de sa démarche philosophique en se recentrant sur les notions d'auteur et de sujet. Au rebours de l'archéologie foucaldienne, où le texte et l'individu importent moins que les configurations épistémologiques et socioculturelles qui les

12. V. Y. Mudimbe, L'Autre Face du Royaume, Lausanne, L'Âge d'Homme, 1973, p. 100 ; L'Odeur du Père, Paris, Présence africaine, 1982, p. 36 [désormais $O P$ ] ; IA, p. 80 et 166. 
rendent possibles, nos trois critiques étudient «l'échange dynamique entre les auteurs individuels et les vastes entreprises politiques formées sur le territoire intellectuel et imaginaire » $(O, 27)$, et ils procèdent ainsi selon une «perspective hybride », à la conjonction des concepts de tradition et d'auteur $(O, 23)$. Croire en «l'influence déterminante d'écrivains individuels sur le corpus des textes et des énoncés, par ailleurs collectif et anonyme, constituant une formation discursive» $(O, 37)$, ou juger, comme Mudimbe, que «le sujet ne saurait être une illusion pure, ou simplement l'ombre d'une épistémè », mais qu' «il prend part à la constitution ou à la modification d'un ordre épistémologique » (IA, 35), évite l'effet de compression d'une démarche strictement archéologique qui encourt le risque de réduire le divers au même. Mais surtout, prêter attention à cette influence, c'est s'offrir les moyens de reconsidérer sérieusement la prise de parole des écrivains de la marge, et c'est donc tenir compte, dans le champ du savoir, des écrivains issus des peuples colonisés par l'Europe et de l'influence qu'ils peuvent exercer, des transformations qu'ils peuvent initier dans la configuration épistémologique. Ce qui caractérise en effet la prise de parole de «l'Autre », c'est qu'elle se situe également dans l'espace du savoir produit sur l'Afrique ou l'Orient: savoir dont il est l'objet et dont il se veut avant tout le sujet, cherchant à se réapproprier l'initiative du discours ; espace du savoir que sa prise de parole cherche donc à reconfigurer, afin de mettre en évidence comment le point de vue occidental s'est généralement conforté, sous couvert de science, dans ses présupposés, à teneur idéologique. Si Mudimbe dénonce l'inféodation fréquente des discours africains à l'hégémonie du discours occidental $(O P, 100-101 ; I A, 87$ et 185), il sait tout autant tirer parti des ruptures, sinon des fissures inhérentes à ce dernier pour promouvoir une stratégie alternative :

Ce serait pur non-sens de reprocher à l'Occident son héritage oriental. Par exemple, personne ne refuserait à Heidegger le droit de philosopher à l'intérieur des catégories du Grec ancien. C'est son droit d'exploiter n'importe quelle part de cet héritage. Ce que par là je veux dire, c'est ceci : la tradition occidentale de la science, aussi bien que le traumatisme de l'esclavage et de la colonisation font partie de l'héritage actuel de l'Afrique. K. Nkrumah a souligné à juste titre, dans son Consciencisme (chapitre 3), que les Africains doivent accepter ces héritages au même titre que les contributions musulmanes et que leur propre passé et leur propre expérience. Bien plus, on pourrait également concevoir les signes intellectuels de l'altérité non point tant 


\section{Maîtrise et déformation: les Lumières diffractées}

comme un projet pour la fondation d'une nouvelle science, mais plutôt comme une manière de réexaminer les cheminements du savoir humain dans un monde de propositions et de choix qui rivalisent les uns avec les autres. Concrètement, sur l'arrière-plan des politiques coloniales de la conversion, ce mode semble impératif. [...] Par-delà aussi bien qu'à l'intérieur d'études comparées et de leur exposition, la logique de ce mode institutionnaliserait une réévaluation des normes, des voix et des consensus antérieurs » (IA, 79-80).

Quant à Mbembe, il s'est très tôt démarqué de ce qu'il appelle « les problématiques usées du nationalisme, de la résistance », pour s'intéresser plutôt aux «modalités de l'insoumission et des usages de la raison »: cette démarche, inaugurée dans son ouvrage sur l'indiscipline des populations africaines face à la domestication entreprise par les missionnaires chrétiens ${ }^{13}$, lui a ensuite permis, dans son étude historique sur La Naissance du maquis dans le Sud Cameroun (1920-1960) ${ }^{14}$, de mettre en évidence «les processus par lesquels les natifs se constituèrent à la fois comme sujets exerçant et subissant des relations de pouvoir et comme agents moraux de leurs actions ${ }^{15} \gg$. En redonnant aux subjectivations toute la primauté qu'elles méritent, Mudimbe et Mbembe mettent l'accent sur les puissances d'agencement et sur la pluralité des positionnements qui rendent possible ce que Michel Foucault appelle « l'espace du savoir », ou ce que Pierre Bourdieu appelle «un champ». C'est d'ailleurs une telle conception du discours, comme « localisation stratégique du sujet ${ }^{16} »$, qui a conduit Mudimbe à préférer, dès l'incipit de son Invention of Africa, la notion de gnosis à celle d'épistémè :

Ce livre a évolué de manière accidentelle, comme le résultat d'une invitation

à préparer un panorama de la philosophie africaine. À strictement parler, la notion de philosophie africaine renvoie aux contributions faites par des Africains qui pratiquent la philosophie à l'intérieur du cadre défini de la disci-

13. Afriques indociles, christianisme, pouvoir et État en société postcoloniale, Paris, Karthala, 1988.

14. Paris, Karthala, 1996.

15. Ibidem, p. 10.

16. «J'utilise simplement cette notion de stratégie», écrivait Said, «pour définir le problème rencontré par tout écrivain traitant de l'Orient : comment l'appréhender, comment l'approcher, comment éviter d'être vaincu ou submergé par sa sublimité, son étendue, ses terribles dimensions. Celui qui écrit sur l'Orient doit définir sa position vis-à-vis de celui-ci : traduite dans son texte, cette localisation comprend le genre de ton narratif qu'il adopte, le type de structures qu'il construit, l'espèce d'images, de thèmes, de motifs qui circulent dans son texte - qui tous s'ajoutent à des façons délibérées de s'adresser au lecteur, de saisir l'Orient et enfin de le représenter ou de parler en son nom » $(O, 33)$. 
pline et de sa tradition historique. Ce n'est que de manière métaphorique, ou au mieux, dans une perspective historiciste, qu'on pourrait étendre la notion de philosophie aux systèmes traditionnels de pensée en Afrique, en les considérant comme des processus dynamiques dans lesquels les expériences concrètes sont intégrées dans un ordre de concepts et de discours. J'ai donc préféré parler de gnosis africaine. [...] Dans ce livre, un cadre plus large semble mieux convenir à l'étendue des problèmes traités, qui tous sont basés sur une question préliminaire : dans quelle mesure peut-on parler d'un savoir africain, et en quel sens ? Étymologiquement, gnosis est lié à gnosko, qui veut dire "savoir" en grec ancien. De manière spécifique, gnosis signifie volonté de savoir, enquête, méthodes de connaissance, investigation, et même connaissance de quelqu'un. Le mot est souvent utilisé dans un sens plus spécialisé, celui d'un savoir supérieur et ésotérique, et il renvoie ainsi à un savoir conventionnel, commun, bien structuré, mais qui se trouve strictement sous le contrôle de procédures spécifiques pour son usage comme pour sa transmission. Gnosis est, par conséquent, différent de la doxa, ou l'opinion, et d'un autre côté ne peut être confondu avec l'épistémè, comprise tout à la fois comme science et comme configuration intellectuelle générale » (IA, ix).

L'épistémè foucaldienne désignait ce qui préexiste aux sujets et à leurs discours ; elle était «ce qui rend possible » leurs positions et leurs énoncés et se trouvait, dans le même temps, une totalité close sur ellemême, puisque le savoir qu'elle proposait ne pouvait se générer qu'en se déclinant, à l'infini, à l'intérieur d'un même cadre, toujours semblablement structuré en dépit de ses variations et de ses ruptures épistémologiques. Ainsi Foucault avait beau distinguer, dans Les Mots et les Choses, plusieurs ruptures et, par conséquent, plusieurs configurations, indexées sur des modèles différents du « savoir »-l'épistémè de la Renaissance l'ordonne selon la ressemblance, celle de l'Âge classique s'articule sur la représentation, et celle qui nous domine depuis le XVIIII siècle se centre sur l'homme, comme sujet producteur, mais aussi comme objet produit par ce même savoir -, il n'en restait pas moins que la « configuration générale », quant à elle, ne variait guère dans ses préoccupations essentielles. On pouvait passer d'une théorie de la richesse à une économie, d'une histoire naturelle à une biologie, et d'une grammaire générale à une philologie, générant ainsi une nouvelle « triade du savoir » dans les interstices de laquelle viendraient se loger toutes les sciences humaines et sociales, un continuum et un principe d'identité n'en continuaient pas moins de traverser les siècles et leurs ruptures - ce continuum étant 
évidemment «l'épistémè du monde occidental» $(M C, 262)$ et ce principe d'identité, " une certaine position de la ratio occidentale qui s'est constituée dans son histoire et qui fonde le rapport qu'elle peut avoir à toutes les autres sociétés, même à cette société où elle est historiquement apparue » $(M C, 388)$. En tant que « rapport fondamental à toute histoire, et qui lui permet de se lier aux autres cultures sur le mode de la pure théorie», ce mode relationnel s'avérait d'emblée relatif, étant «une certaine position de la raison », face à laquelle on pouvait donc envisager d'autres « usages », et d'autres modes relationnels. Or, c'est précisément dans cette marge de manœuvre, ou dans cette possibilité d'une alternative que nous devons situer la notion de « gnosis africaine » élaborée par Mudimbe. En «préférant » cette notion à celle d'épistémè, le philosophe africain rétablit avant tout la subjectivité dans ses droits et dans ses devoirs, c'est-à-dire dans ses « usages de la raison ». La gnose s'apparente en effet à une démarche personnelle et volontaire, c'est-à-dire à l'activité réflexive que tout cogito peut opérer sur lui-même et sur son rapport au monde, mais également aux autres : la dimension relationnelle est directement impliquée dans la notion de gnosis, qui peut également désigner - et Mudimbe prend soin de le noter - une connaissance de l'autre, ou du moins une certaine «familiarité », voire une intimité. La notion de gnosis laisse ainsi entrevoir un autre modèle relationnel, qui ne serait plus, cette fois, "sur le mode de la pure théorie », mais bel et bien un savoir empirique, né de la relation concrète et quotidienne avec «l'autre » comme «familier» (au sens originel et latin, l'adjectif familiaricus et le nom propre familiaris désignant l'appartenance à une même famille), et non plus comme « exotique », comme étrange ou « étranger »- au sens grec du xénos ou du barbaros. La gnose doit également s'envisager dans une perspective interculturelle: il n'y a aucune racialisation dans l'adjonction de son prédicat, mais simplement la mention d'un ancrage historique et culturel singulier - dans la réalité « africaine » contemporaine et ses mémoires conflictuelles - qui ne tolère nul essentialisme et ne confère aucun droit de parole réservée : nul Africain ne possède, pas plus qu'aucun Occidental, de droit exclusif à parler au sujet ou au nom du monde noir, puisque cela appartient à tout individu qui choisit de s'exprimer à partir de ce « phénomène de l'acculturation » que Mudimbe décrit comme une « figure du métissage, - c'est-à-dire comme une hybridation culturelle qui témoigne des dynamiques contemporaines des dialogues entre les peuples et les histoires » (TF, XII). L'inscription 
dans cet « espace intermédiaire entre la prétendue tradition africaine et la modernité projetée du colonialisme » $(I A, 5)$, constitue alors ce qu'on pourrait appeler la « condition postcoloniale » :

Dans le cas de l'Afrique de l'Ouest, [...] le bloc des traditions supposément traditionnel [...] était en réalité un monument de cultures se mélangeant entre elles au point que leurs propres genèses étaient toujours déjà plurielles. [...] Dans la reconfiguration socio-économique et culturelle de l'Afrique au XIX ${ }^{\mathrm{e}}$ et au début du $\mathrm{XX}^{\mathrm{e}}$ siècles, un espace métissé s'impose de lui-même contre les anciennes traditions qui sont loin d'être pacifiques et le nouveau programme qui veut se substituer à elles dans l'histoire coloniale. Il n'est donc point étonnant que la conscience produite par ce nouvel espace remette parfois en question la validité de ce qui est présenté comme la vérité et, sous la confluence des courants qui l'ont rendue possible, recherche la vérité cachée, supposément ésotérique, de son propre passé ou bien cherche à découvrir le paradigme secret qui explique la puissance de la volonté de vérité occidentale (TF, 152-154).

Si Mbembe exprime, quant à lui, son exaspération à l'égard des psittacismes «qui n'en finissent plus de tout problématiser en termes d'“invention" et d'"hybridation", de "fluidité" et de "négociation des identités" » $(D P, 14)$, il s'accorde avec Mudimbe pour mettre en relief la multiplicité des historicités et des imbrications culturelles en Afrique, et il veut alors prioritairement penser leur interpénétration avec d'autres temporalités et d'autres configurations.

La légalité propre des sociétés africaines, leurs propres raisons d'être et leur rapport à rien d'autre qu'à elles-mêmes s'enracinent dans une multiplicité de temps, de rythmes et de rationalités qui, bien que particuliers et, parfois, locaux, ne peuvent pas être pensés en dehors d'un monde qui s'est, pour ainsi dire, dilaté. D'un strict point de vue de méthode, ceci signifie qu'à tout le moins à partir du XVe siècle, il n'y a plus d'“historicité propre" de ces sociétés qui ne soit, elle-même, encastrée dans ces autres temps et rythmes que conditionna largement la domination européenne. Du coup, traiter de leur légalité propre ne consiste pas seulement à rendre compte de ce qui se noue, sur le continent même, à l'interface entre le travail des forces internes et celui des acteurs internationaux. Cela suppose une plongée critique dans l'histoire occidentale elle-même et dans les théories qui prétendent l'interpréter $(D P, 21)$. 
La condition postcoloniale devient ainsi un rapport d'action réciproque : elle désigne tout à la fois l'incidence occidentale sur l'histoire mondiale, et la présence ou l'influence des non-Occidentaux dans l'histoire des sociétés occidentales - ou ce que Mbembe a récemment appelé « l'histoire de notre présence au monde et de la présence du monde en notre $\operatorname{sein}^{17} »$.

«Penser de manière critique la postcolonie » implique deux impératifs. Il faut d'abord tenir compte des durées multiples qu'elle renferme, et qui sont « faites de discontinuités, de renversements, d'inerties, d'oscillations qui se superposent, s'enchevêtrent et s'enveloppent les unes les autres » $(D P, 34)$; et il faut ensuite "s'interroger sur le passage de l'un (l'avant la colonisation) à l'autre (l'après la colonisation) et sur la question du transit que ce passage pose » $(D P, 36)$. Si la réflexion postcoloniale ne peut se déployer hors d'une inscription « dans la longue durée », elle ne saurait pas davantage s'abstraire d'une préoccupation centrale pour « la thématique du sujet africain en train de surgir » à travers ces divers processus du transit et du déplacement. La subjectivité, parce qu'elle doit elle-même « s'envisager comme temporalité » $(D P, 34)$, constitue en définitive le vrai pivot de cette action réciproque qui définit la condition postcoloniale, et l'axe véritable du « basculement des mondes » qu'elle met en branle (DP, 20).

\section{L'historiographie française, entre pari postcolonial et relaps colonial}

Dans l'inspiration d'Achille Mbembe, un nombre croissant d'historiens et de politologues français ou francophones se réclament aujourd'hui des études postcoloniales. Certains soulignent même, dans un livre qui se veut un manifeste autant qu'un brûlot, « l'urgence à transformer les interrogations, les analyses, les études sur la période coloniale et postcoloniale, dispersées jusque-là, en un mouvement, une "école" de recherche à l'image de ce qui s'est passé aux États-Unis, en Asie, en Inde, en Afrique, en Angleterre autour des "postcolonial studies"18». Parce que

17. « La République et l'impensé de la "race" », op. cit., p. 149.

18. Nicolas Bancel, Pascal Blanchard, Françoise Vergès, La République coloniale, essai sur une utopie, Paris, Albin Michel, collection Idées, 2003, p. 7 [RC]. 
«l'histoire coloniale en France » aurait «"loupé le coche" de bon nombre de questions historiques qui font actuellement le miel des études contem-

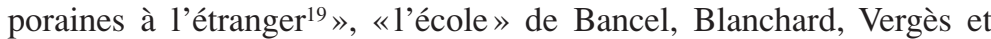
Lemaire s'évertue à prendre le train postcolonial en marche, mais on peut se demander si leurs publications motivées par «l'urgence» ne sont pas trop hâtives pour être vraiment fidèles aux exigences de cette nouvelle approche critique. Car il ne suffit pas d'avoir la formule pour maîtriser la démarche, et le mot peut d'autant mieux masquer certains manquements que son sens est lui-même équivoque ${ }^{20}$. Dans les textes de Bancel, Blanchard, Lemaire et Vergès, cette ambivalence remplit de fait une fonction cruciale, puisqu'en dépit des déclarations d'intention le sens objectif prime souvent sur le sens subjectif. Ce n'est donc point tant la démarche qui importe que le moyen de construire un balisage historique $^{21}$. Quoiqu'ils s'en défendent ${ }^{22}$, leur vision historique ne cesse, d'un côté, d'insister sur la prégnance, voire la préséance d'une « culture coloniale » dans les réalités postcoloniales, et elle tend ainsi à « faire du colonialisme le marqueur déterminant de l'histoire », tandis que, de l'autre, elle laisse supposer un avantage sinon une supériorité des cultures postcoloniales, et elle tend alors à « retomber dans le schéma linéaire du temps propre à l'Europe, marqué par la téléologie du "progrès" et de la "civilisation" » comme l'a très bien souligné, dans un autre contexte, le critique littéraire Jean-Marc Moura ${ }^{23}$. Sur un autre plan, cette « école de recherche » prend soin de placer sous le signe de la multiplicité « les phénomènes engendrés par le fait colonial », tels qu'ils se sont «poursuivis, mais aussi métissés, transformés, résorbés, reconfigurés » $(F C, 12)$, ou bien elle souligne que «la société française est aujourd'hui traversée par des processus de transformations protéiformes » $(F C, 28)$, que la République demeure un «projet évolutif et [une] réalité historique changeante, soumise à des transformations » $(R C, 13)$; mais parallèlement

19. Nicolas Bancel, «L'histoire difficile : esquisse d'une historiographie du fait colonial et postcolonial », dans Nicolas Bancel, Pascal Blanchard, Sandrine Lemaire (éd.), La Fracture coloniale. La société française au prisme de l' héritage colonial, Paris, La Découverte, 2005, p. 84 [FC].

20. Voir supra, l'entretien avec Anne Berger.

21. Voir notamment l'essai de Nicolas Bancel dans La Fracture coloniale.

22. «Sans prétendre faire de ce passé colonial l'épicentre de nos tourments contemporains, il nous faut admettre que l'héritage n'est pas sans séquelles » $(R C, 16)$; « Nous n'entendons aucunement réduire l'éclairage de la société française contemporaine aux seules lumières des articulations colonisation/postcolonie - les nier reviendrait, à l'inverse, à s'interdire d'en saisir des dimensions essentielles » $(F C, 12)$. 23. Littératures francophones et théorie postcoloniale, Paris, Puf, 1999, p. 4. 
elle réactive des raisonnements simplistes et univoques, en identifiant systématiquement l'idée républicaine à une « utopie coloniale » $(R C, 13)$, ou en ne reconnaissant dans la relation coloniale qu'une situation de domination, sans tenir compte des alternatives relationnelles et de la redistribution des pouvoirs qui se sont également élaborées durant l'ère coloniale. De ces diverses tensions s'ensuit un obscurcissement théorique qui contamine les notions centrales de «république coloniale» et de «fracture coloniale ${ }^{24} »$. Mais on peut surtout regretter une occultation complète des alternatives ou des actions juridiques, politiques, que les intellectuels ou les représentants des ex-colonies ont proposées et opposées à ce que nos auteurs appellent la « République coloniale». Bancel et Blanchard s'évertuent, par exemple, à prôner une « complexification des perspectives historiques, l'histoire coloniale pouvant être envisagée comme un processus dialectique, qui engage des transformations croisées dans les colonies et la métropole » $(F C, 85)$; «rares sont ceux», ajoutent-ils, « qui aujourd'hui encore prennent la colonisation du côté de ses effets en métropole ». Tellement rares, en effet, que ces historiens en rupture de ban oublient eux-mêmes de considérer les démarches historiques entreprises par des hommes politiques noirs (africains, antillais) pour faire éclater le système colonial et parvenir à une pleine actualisation de l'idée républicaine. Seul Aimé Césaire trouve grâce à leurs yeux, pour avoir été l'un des premiers à exposer la logique de domination inhérente à tout pouvoir colonial $(R C, 47)$, mais quid de Blaise Diagne, de Félix Éboué, de Gaston Monnerville, de Lamine Gueye, de Doudou Thiam? Éluder ceux-là mêmes qui furent les premiers à plaider pour une justice semblable et une égalité effective, ou qui luttèrent et œuvrèrent pour la plus grande «portée de la citoyenneté française dans les territoires d'outre-mer ${ }^{25}$ », c'est non seulement faire l'économie de certains processus majeurs, qui introduisent au cœur d'une véritable «histoire africaine de la France ${ }^{26} »$, mais c'est également reproduire le geste colonial, lequel tendit toujours à minorer ou mépriser les actions dont l'effectivité historique visait précisément à l'éradiquer par la racine. Bancel, Blanchard, et Vergès se contentent de rappeler que « les colonisés retour-

\footnotetext{
24. Voir, dans ce numéro, la note de lecture que propose Grégoire Leménager.

25. Selon le titre de la thèse de Doudou Thiam pour le doctorat de droit : La Portée de la citoyenneté française dans les territoires d'outre-mer, Paris, Société d'études africaines, 1953.

26. Ainsi que l'a très bien rappelé Bernard Mouralis dans son ouvrage exemplaire, République et colonies, entre histoire et mémoire, Paris, Présence africaine, 1999.
} 
nèrent le discours républicain contre le discours colonial républicain pour développer des projets émancipateurs » $(R C, 14)$, sans jamais nommer personne et sans plus de précisions; ou bien ils éludent systématiquement la période 1945-1946, au cours de laquelle s'opéra pourtant, « en liaison directe avec le rôle joué par l'Afrique au cours de la Seconde Guerre mondiale, la transformation du système colonial ${ }^{27}{ }^{2}$; enfin ils déplorent qu'on ne trouve généralement « aucun auteur africain, ni maghrébin, ni de l'ex-Indochine » dans les publications ou revues consacrées « au temps des colonies » $(R C, 148)$ mais ils négligent au passage qu'on pourrait en dire presque autant de leur propre bibliographie. S'ils voient aujourd'hui, dans l'actualité pressante de la question coloniale, le «symptôme d'un "retour du refoulé"» $(F C, 10)$, on pourrait tout aussi bien reconnaître, dans leur indifférence à l'égard des agencements de sujets éminemment politiques et politisés, la persistance d'un refoulement, ou à tout le moins d'une occultation. La colonie a certes pu servir de « laboratoire » pour des logiques de domination qui ont trouvé, ensuite, à s'exercer sur le territoire métropolitain, et « les représentations de l'Autre », constitutives et dérivées de la relation esclavagiste, puis de la relation coloniale, trouvent certes à s'exprimer encore de nos jours de manière à peine déguisée; mais dans le même temps, certaines conditions historiques, sociologiques et idéologiques (au premier rang desquelles on trouve justement l'idée républicaine), ont d'emblée fragilisé le système de domination et rendu possible sa contestation croissante, tandis que les rapports des métropolitains aux ressortissants des (ex-) colonies ne furent pas toujours uniquement nourris par les stéréotypes, mais ont pu également reposer sur « une connaissance mutuelle, imparfaite sans doute », voire sur « une familiarité entre Français et Africains ${ }^{28} »$; enfin, et ce n'est pas la moindre chose, les « raisons d'agir » des hommes et des femmes issus des territoires d'outre-mer ont pu affecter et altérer de manière positive les logiques sociales et politiques en métropole. Il faut donc, si l'on veut sérieusement mesurer l'influence des " effets en retour », accorder une plus grande attention aux agencements de celles et ceux qui furent, somme toute, les premières victimes du « racisme républicain », et il est impératif, si l'on veut apprécier la complexité et la multiplicité des formes relationnelles entre république et colonies, colonisation et postcolonie,

27. Ibid., p. 34.

28. Ibid., p. 39. 
de tenir également compte de tout ce qui s'écarte, justement, des catégorisations faciles et simplistes de l'altérité. La littérature, la presse, le cinéma peuvent notamment désamorcer autant que nourrir un imaginaire de la stéréotypie. J'ai étudié ailleurs comment certains écrivains et hommes d'action antillais et africains-américains avaient, les premiers, exposé les contradictions inhérentes au colonialisme républicain, et j'ai pu montrer que leurs positionnements préfiguraient non seulement les débats que nous poursuivons aujourd'hui, mais surtout aidaient à dépasser les jugements de valeur ou les apories critiques sur les réalités historiques de «la République coloniale ${ }^{29} »$. Je voudrais à présent formuler, en guise de conclusion, quelques propositions pour mieux apprécier l'importance et l'influence des écrivains et intellectuels noirs dans notre paysage épistémologique, mais aussi culturel, social et politique.

\section{Lumières noires, discours marron}

Il nous faut d'abord, selon moi, prioritairement et impérativement étudier dans quels contextes - institutionnels, épistémologiques, historiques et socioculturels - et selon quels modes spécifiques la figure de «l'intellectuel noir» est apparue en Occident, pour s'imposer progressivement dans le champ des discours et des disciplines qui constituent notre épistémè actuelle. Ce faisant, nous devons surtout retracer comment les écrivains et penseurs noirs (qu'ils soient américains, antillais ou africains) se sont situés par rapport aux discours dominants, et notamment ceux qui portaient sur le monde noir - que ces discours soient ouvertement « littéraires » (récits de voyage, fictions) ou délibérément « scientifiques », comme les discours philosophiques, historiques, anthropologiques ou sociologiques. S'ils n'acceptent pas sans réserve le savoir constitué par les maîtres du discours occidental, ils ne le rejettent pas non plus intégralement. En portant notre attention sur les premières figures d'intellectuel(le)s noir(e)s - par là je désigne les hommes et les femmes qui ont précisément revendiqué cette qualité, et conçu leur discours comme une tradition distincte de l'ordre dominant -, on peut identifier une genèse explicite au tournant des $\mathrm{XIX}^{\mathrm{e}}$ et $\mathrm{XX}^{\mathrm{e}}$ siècles, dans les contextes améri-

29. Anthony Mangeon, « République et colonies : l'éclairage des Lumières noires (Alain Leroy Locke, René Maran, Félix Éboué) », Francophone Postcolonial Studies, IV-2, Londres, 2006. 
cains, antillais puis africains, et l'on peut également relever certaines symétries entre les diverses personnalités qui ont joué un rôle prépondérant dans l'émergence et la construction d'une « conscience noire ». Qu'il s'agisse d'Africain(e)s-Américain(e)s comme William E. B. Du Bois, Jessie Fauset, Alain Leroy Locke, Zora Neale Hurston, d'Antillais(e)s comme Claude McKay, Paulette et Jane Nardal, Aimé et Suzanne Césaire, C. L. R. James, Eric Williams, ou d'Africains comme $\mathrm{Pa}$ Ka Isaka Seme ou Léopold Sedar Senghor, pour ne citer que quelques noms, on peut d'emblée découvrir qu'elles/ils ont toutes et tous en partage ce que Du Bois appelait une « double conscience ${ }^{30}$ » (sociale, linguistique, culturelle) ainsi qu'une formation intellectuelle diversement interdisciplinaire et transnationale, à la croisée de plusieurs disciplines et sur plusieurs continents. Ce sont des traits notoires qu'on retrouvera plus tard chez d'autres éminentes figures, comme Franz Fanon, Édouard Glissant, Maryse Condé, Buchi Emecheta, Françoise Ugochukwu, Valentin-Yves Mudimbe, Achille Mbembe, Alain Mabanckou, etc. Pardelà ces données conjoncturelles, on peut surtout observer un triple processus.

1. L'émergence de l'intellectuel noir, comme nouvelle figure de discours manifeste un changement de perspective et d'attitude qu'on peut traduire par le passage d'un oxymore à un paradoxisme : on ne pouvait être en effet noir et intellectuel dans la doxa communément admise en Amérique et en Europe ; mais à compter de Du Bois et de Locke, la figure de l'intellectuel noir se construit et se vit comme une double postulation, puisqu'on veut être le plus intellectuel et le plus cosmopolite possible, c'est-à-dire guidé par la plus grande exigence de rationalité et d'universalité, tout en demeurant le plus nègre possible, c'est-à-dire le mieux ancré dans des traditions culturelles singulières, où la conscience de soi fut aussi fondée et transformée par une double expérience d'aliénation : la traite, puis la colonisation.

2. Le développement du discours noir, en tant qu'acte d'énonciation et en tant qu'ensemble d'énoncés plus ou moins constants, est le produit d'une double stratégie. Il y a, d'une part, maîtrise de la forme, c'est-àdire compréhension rationnelle des formes de relations (sociales, culturelles, économiques, politiques) historiquement constituées entre l'Europe

30. The Souls of Black Folk (1903) ; Les Âmes du peuple noir, traduction, annotation et postface de Magali Bessone, Paris, Éditions Rue d'Ulm, 2004, p. 10-11. 
et le monde noir, mais aussi appropriation des langues, des genres littéraires, des pratiques discursives et des langages conceptuels dominants (philosophie, sociologie, anthropologie). Cette maîtrise de la forme est, d'autre part, le moyen d'une déformation constante de la maitrise ${ }^{31}$, opérant en effet un réagencement des rapports de force et une dislocation du Pouvoir/Savoir exercé par le monde européen sur le monde noir, au bénéfice de nouveaux rapports culturels, d'une nouvelle économie discursive et d'un nouvel ordre du savoir. Le discours noir est donc le lieu d'un véritable marronnage, qui cherche à échapper aux structures coloniales et capitalistes en se reterritorialisant hors d'une Afrique mythique et prétendument traditionnelle, dans une Amérique fondamentalement pluraliste et multiculturelle, ou dans des Indes occidentales fortement marquées par la présence du Tout-Monde et la créolisation des cultures.

3. L'indiscipline du sujet noir, son refus de s'enfermer dans une nature et sa volonté d'être un projet ont ainsi engagé l'articulation de sa parole à la croisée des genres discursifs (littérature, philosophie, sciences humaines et sociales) et dans le dialogue des cultures (africaines et européennes). Le dessein fondamental d'une telle indiscipline est, en définitive, d'accompagner un processus majeur : la transformation progressive de notre épistémè occidentale en une épistémè plurielle, diffractée, travaillant mais aussi travaillée par d'autres épistémai.

Au rebours de Michel Foucault, qui a théorisé la transformation épistémologique sur le seul mode de la rupture, et qui la situait prioritairement au niveau des sciences, et notamment des sciences de l'homme, il faut d'abord souligner l'importance déterminante de la littérature dans l'ordre du savoir, et il faut ensuite montrer comment une épistémè est avant tout relationnelle, ou comment elle se construit aussi dans un rapport dialogique avec d'autres configurations. La transformation doit dès lors s'envisager sur un mode continuiste, inscrit au sein même des relations historiques entre les peuples et les cultures ; quant à l'émergence du discours noir, parce qu'elle est avant tout indiscipline et marronnage, elle laisserait peut-être entrevoir une reformulation des Lumières, ou un nouvel âge interracial et interculturel que j'ai proposé ailleurs d'appeler les Lumières noires.

31. J'emprunte cette paire de concepts au critique africain-américain Houston Baker Jr, Modernism and the Harlem Renaissance, Chicago, University of Chicago Press, 1987, p. XVI et 75. 
On peut finalement affirmer que sur les plans historique, culturel et institutionnel, le discours noir s'ancre largement à l'intérieur de l'épistémè occidentale, mais qu'il s'y est développé à la faveur d'une disruption majeure qui la traverse et la perturbe, depuis des siècles, alors qu'on la présente habituellement comme uniforme et univoque. On peut ainsi, sans trahir ses exigences, interpréter le discours noir comme la continuation la plus effective d'une longue discontinuité qui remonte, à tout le moins, à l'âge baroque, et qui me semble, depuis près d'un siècle à présent, avoir définitivement accéléré la transformation de l'épistémè occidentale en une épistémè diffractée. Et loin de nous acheminer vers une disparition de l'homme et de l'esprit des Lumières, ainsi que le souhaitait ou le prophétisait Michel Foucault, cette « continuation d'une discontinuité » bénéficie, précisément, de l'avènement et du développement des sciences de l'homme depuis le XVIII $I^{\mathrm{e}}$ siècle, puisqu'elle se conçoit fondamentalement comme un anthropou-logos, ou un « discours par un sujet » qui ne serait désormais plus univoque ou monologique, mais assurément dialogique et polyphonique. Cette énonciation nouvelle et concurrentielle fait de surcroît entrer la littérature en dialogue avec les sciences de l'homme, et notamment l'histoire et la philosophie, supprimant par là les statuts privilégiés que Foucault cherchait à leur préserver, si bien que, dans les trois domaines majeurs qui ont étudié l'homme en tant qu'être vivant, parlant, et travaillant, on peut désormais découvrir une reconfiguration complète, voire de singuliers renversements généalogiques ${ }^{32}$.

La réalité de «l'homme, être vivant » n'est plus seulement étudiée selon des modèles biologiques, mais grâce à la détermination progressive d'une nouvelle anthropologie qui, désormais, procède et découle de la phénoménologie, après avoir débarrassé cette dernière de sa tentation transcendantale et de sa posture gnoséologique. On peut relever d'autres constantes du discours noir en tant qu'anthropou-logos lorsqu'on observe les filiations que les penseurs noirs ont établies avec l'anthropologie culturelle, ou bien avec les sociologues qui ont prioritairement étudié les formes de socialisation et les conflits qu'elles peuvent engendrer. Mais qu'il s'agisse des comparaisons qu'on peut établir entre, par exemple,

32. Je reprends, à partir d'ici, les principales conclusions de ma thèse : Lumières noires, discours marron, indiscipline et transformations du savoir chez les écrivains noirs américains et africains, itinéraires croisés d'Alain Leroy Locke, V. Y. Mudimbe, et de leurs contemporains (décembre 2004, université de Cergy-Pontoise). 
Alain Locke et ses inspirateurs (Franz Boas, Gabriel Tarde et Georg Simmel) ou inversement, les penseurs américains et européens qu'il a directement influencés (dont Melville Herskovits et Michel Leiris), ou qu'il s'agisse des liens qu'on peut reconnaître entre Valentin-Yves Mudimbe et des penseurs plus contemporains comme Claude LéviStrauss et Pierre Bourdieu, ces diverses « parentés » me semblent aller dans le même sens - c'est-à-dire vers une critique de l'évolutionnisme linéaire et de l'ethnocentrisme irréfléchi, ce dernier se trouvant d'autant plus douteux et dangereux qu'il est déterminé, sur le plan historique, par de contingentes circonstances de domination sociale, culturelle ou politique. En somme, même au niveau des sciences humaines et sociales, le discours noir s'est toujours stratégiquement allié aux penseurs occidentaux qui se trouvaient être les critiques les plus féroces de l'hégémonisme des classes dominantes ou de l'impérialisme des peuples européens. Mais en indexant sa réflexion humaniste sur le strict plan de «l'expérience », et en réorientant son étude des « rapports intersubjectifs » ou du « contact culturel » vers leurs dimensions « interraciales » et « interculturelles », l'intervention du discours noir me semble avoir été cruciale, dans la mesure où elle a toujours favorisé et poursuivi une critique conjointe de l'essentialisme et du naturalisme intransigeant qui ont longtemps dominé les discours sur l'homme-en-tant-qu'être-vivant - y compris, parfois, chez les alliés les plus « objectifs » des écrivains et penseurs noirs.

Passons à présent au rapport au langage, c'est-à-dire aux réflexions sur l'homme-en-tant-qu'être-parlant. Là encore, on peut découvrir, au sein du discours noir, la même articulation entre philosophie et anthropologie, ainsi qu'une semblable filiation avec le régime turbulent de la pensée occidentale, et tout particulièrement avec la philosophie de Johann Gottfried Herder. La pratique africaine de la philosophie, dans ses tensions conflictuelles avec l'anthropologie, me semble avoir plus spécifiquement renoué avec l'attention qu'accordait Herder à l'influence des langues sur les configurations ou les Weltanschauungen culturelles ; le programme littéraire des Africains-Américains ou celui des Antillais relèvent davantage, quant à eux, des problématiques du nationalisme culturel comme thésaurisation des formes populaires et perpétuation du Volksgeist ; mais ces orientations m'apparaissent, dans leurs divergences mêmes, comme les deux versants complémentaires d'une même logique conceptuelle. Celle-ci poursuit en effet, sur le plan des relations entre langage et expérience, les renversements généalogiques qu'on peut obser- 
ver au niveau de l'homme-en-tant-qu'être-vivant. À la critique de l'essentialisme, le discours noir et ses alliés pragmatistes ajoutent donc une critique du représentationnalisme qui débouche nécessairement sur une recomposition du discours. La connaissance n'y est plus pensée sur le mode spéculaire de la correspondance, mais sur celui de la cohérence, et le savoir cesse d'être indexé sur le modèle sensoriel de la vue pour se penser sur le modèle de l'ouïe, puisque ce sont désormais la continuité et la mise en relation des propositions qui produisent le sens. On privilégie dès lors la « conversation », c'est-à-dire la polyphonie et la perpétuelle rethématisation dialogique. C'est ainsi qu'on peut identifier, inhérentes au développement de la littérature africaine-américaine, une conception et une modélisation musicales du discours qui, de fait, peuvent clairement apparaître comme des « africanismes » lorsqu'on les confronte à la conception et à l'usage de la parole qui président à de nombreuses sociétés d'Afrique de l'Ouest ou d'Afrique centrale, chez par exemple les Malinkés dont les romans d'Ahmadou Kourouma nous content l'histoire, ou chez les peuples dont les ouvrages de Félix Éboué nous ont dressé l'ethnographie ${ }^{33}$, et dont les musiques et littératures congolaises actuelles sont d'évidentes héritières. Certaines anthologies majeures de la littérature nègre - The New Negro (1925), The Negro Caravan (1942), L'Anthologie de la nouvelle poésie nègre et malgache d'expression française (1948), furent elles-mêmes conçues par leurs éditeurs respectifs (Alain Locke, Sterling Brown, Léopold Sedar Senghor) sur un évident modèle d'orchestration polyphonique et de variations thématiques. Par-delà les nouveaux agencements et les nouvelles pratiques littéraires auxquels ces « africanismes » peuvent donner naissance, il me semble également important de noter une confluence avec les conceptions musicales de la parole qu'on peut, par exemple, découvrir chez Cyrano de Bergerac ou chez Jean-Jacques Rousseau, et qui m'apparaissent caractéristiques du régime conceptuel turbulent qui traverse l'épistémè occidentale. Ainsi, dans son interprétation et sa pratique de l'homme-en-tant-qu'être-parlant, le discours noir participe également à la transformation progressive de notre ordre du discours en une configuration plurielle et polyphonique.

33. Voir La Clef musicale des langages tambourinés et sifflés (1935), dont certaines pages furent reprises dans La Revue du Monde noir (6, 1932), puis dans le Bulletin de la Société des recherches congolaises (28, décembre 1941). 
La troisième dimension, qui concerne l'homme comme producteur de richesses matérielles ou bien l'homme-en-tant-qu'être-qui-travaille, est sans doute celle qui fut longtemps la plus étudiée, puisque leur situation historique et leurs préoccupations géopolitiques ont souvent conduit les principaux écrivains et penseurs noirs à contribuer à une critique très élaborée du capitalisme.

Sur tous ces plans qui concernent l'homme en tant qu'être vivant, parlant et travaillant, on peut non seulement retrouver la stratégie caractéristique du discours noir comme "maitrise de la forme et déformation de la maîtrise», mais son indiscipline discursive et son marronnage conceptuel ont, de surcroît, conféré à la littérature un rôle tout aussi déterminant qu'à la philosophie ou aux sciences humaines : il est ainsi permis d'observer comment la littérature peut être engagée sans être idéologique ni dogmatique, comment elle peut être l'expression de singularités historiques et culturelles sans être dépourvue d'universalité, et comment elle participe aux distributions et tribulations du savoir sans pour autant devenir la tribune privilégiée d'un courant unique ou d'une seule école de pensée. Mais un tel constat en appelle nécessairement un autre: si le discours noir, dans son indiscipline et son hybridation des pratiques discursives, participe d'une transformation de notre épistémè, il faut impérativement, sur le plan méthodologique, que la critique qui se penche sur lui agisse de même, et selon les mêmes codes transdisciplinaires et transculturels. En ce sens, c'est bien la critique postcoloniale, en tant qu'elle relève du comparatisme, qui m'apparaît aujourd'hui la mieux armée pour accomplir cette tâche. 